\title{
Rôle du secteur privé dans l'offre de services de santé au Sénégal
}

Population Council

Follow this and additional works at: https://knowledgecommons.popcouncil.org/departments_sbsr-rh

Part of the Demography, Population, and Ecology Commons, Family, Life Course, and Society Commons, Health Policy Commons, and the International Public Health Commons How does access to this work benefit you? Let us know!

\section{Recommended Citation}

Population Council. 2010. "Rôle du secteur privé dans l'offre de services de santé au Sénégal," brief. Dakar: Population Council. 
Les prestataires privés sont souvent d'importants pourvoyeurs de produits et services de santé, et pourtant la contribution du secteur privé est trop souvent non pris en compte dans les initiatives et statistiques de santé publique. Le secteur privé est vaste et diversifié et comprend des prestataires de soins de santé à but non lucratif, confessionnels, et à but lucratif, allant des grands hôpitaux de soins tertiaires aux petites infirmeries. Au Sénégal, les prestataires privés sont une source majeure dans la fourniture de services de santé, offrant près de $22 \%$ des services de contraception (EDS, 2005). Le partenariat avec le secteur privé constitue un élément clé de la stratégie du Ministère de la Santé pour la réalisation des objectifs nationaux de santé.

De solides partenariats entre les secteurs public et privé augmentent la couverture, l'utilisation et la qualité des services et se traduisent par une meilleure satisfaction pour les usagers. Les prestataires de soins de santé privés offrent aux populations toute une gamme de services préventifs et curatifs. Les programmes en milieu de travail où les entreprises privées investissent dans l'offre de services de santé pour leurs employés sont un autre mécanisme de l'engagement du secteur privé dans les efforts nationaux en faveur de la promotion de l'accès des populations aux services de Santé.

\section{Les partenaires bilatéraux du secteur privé du}

\section{Sénégal}

Le projet SMNI / PF / PALU est un projet bilatéral quinquennal de I'USAID visant à favoriser la prestation de services de haute qualité, et à construire de solides liens entre tous les niveaux du système de santé et les communautés, notamment entre le secteur privé et le secteur public. Dans les régions de Dakar et Saint Louis, vingtcinq entreprises privées ont signé un mémorandum d'entente avec l'administration décentralisée des services de santé (les régions médicales) et ont accepté de se joindre ainsi au réseau de prestation de services de santé. Ces entreprises relèvent de deux catégories: 1) les prestataires privés de soins de santé, et 2) les entreprises privées qui fournissent des services de santé pour leurs employés et leurs familles. Certaines de ces entreprises offrent aussi

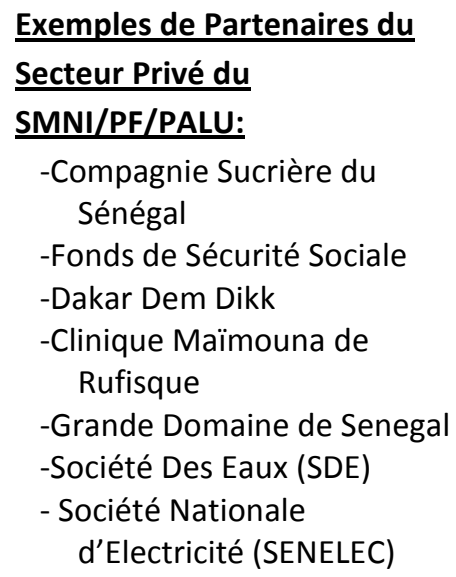

des services aux populations environnantes, même si cela ne fait pas partie pas de leur objectif premier (ex: cas de la Compagnie Sucrière du Sénégal de Richard Toll, du Port Autonome de Dakar, des Industries Chimiques du Sénégal). Construire un réseau entre ces prestataires privés et le secteur public permet d'atteindre les objectifs suivants: améliorer la qualité des soins offerts dans le secteur privé; superviser la fourniture des soins dans le secteur privé, et harmoniser les procédures des secteurs public et privé (notamment la transmission de données au système national d'information sanitaire, et l'amélioration de la chaîne d'approvisionnement national des produits et équipement de santé).

\section{Mise en œuvre de l'initiative du secteur privé}

Dans le cadre du projet SMNI / PF / PALU, chaque entreprise privée a un agrément validé par un protocole d'accord (PA). Le protocole d'accord définit les rôles et responsabilités des acteurs du secteur public et privé et les modalités permettant aux partenaires de collaborer et soutenir efficacement les intérêts de chacun. Certaines entreprises privées ont investi dans des activités de renforcement des capacités des prestataires, tandis que d'autres ont concentré leurs efforts sur des campagnes de sensibilisation en matière de santé de la reproduction (SR) dans les communautés.

Les activités de formation publiquement soutenues peuvent améliorer la qualité et la consistance des services du secteur privé. Grâce au projet SMNI / PF / PALU, 52 prestataires privés de soins de santé ont reçu une formation sur le "paquet de services de planification familiale", comprenant I'orientation sur les Politiques nationales, les Normes et Protocoles (PNP) de SR, les systèmes logistiques et de gestion des produits contraceptifs, et l'utilisation des supports de gestion de l'information sanitaire. Des aide-mémoires, des supports visuels de l'information, d'Education et de Communication (IEC) ainsi que du matériel médical (des kits d'insertion/et de retrait du DIU et des implants) ont été remis aux prestataires du secteur privé après leur formation pour leur permettre d'offrir des services PF de qualité. 
Les activités de plaidoyer et d'éducation sur la PF menées par certaines entreprises du secteur privé ont aussi contribué à la réalisation des objectifs du Ministère de la santé publique. Durant l'année 2009, le projet SMNI / FP / PALU a apporté un soutien à la tenue de trois "Journées Portes Ouvertes" dans des entreprises privées, afin de sensibiliser les populations sur la planification familiale et de fournir des services de santé de la reproduction au sein de leurs communautés. Par exemple, au cours de la Journée Portes Ouvertes organisée à la Compagnie Sucrière Sénégalaise de Richard Toll, 818 préservatifs masculins, 16 préservatifs féminins ont été distribués ; 600 personnes ont reçu des messages d'IEC sur la planification familiale, 44 femmes ont accepté une méthode

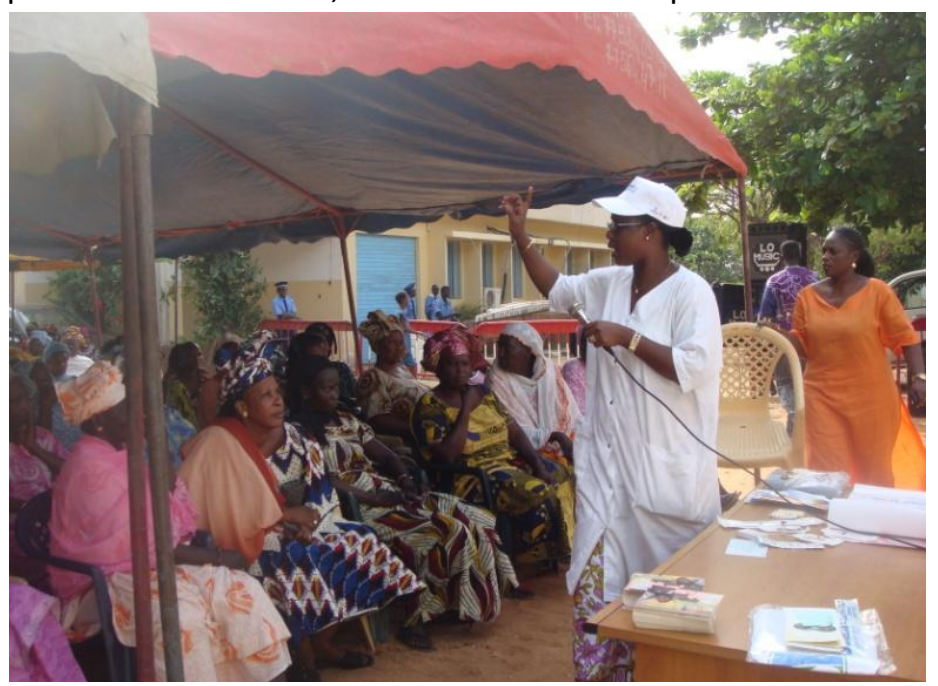

Séance de sensibilisation sur la PF lors des JPO de la CSS

contraceptive autre que les condoms (dont 15 méthodes à long terme); 515 femmes ont eu à bénéficier gratuitement des consultations gynécologiques, et 31 femmes ont bénéficié d'un test de dépistage du cancer du col de l'utérus. Au cours des Journées Portes ouvertes organisées au niveau des ICS de Mboro, 1033 personnes ont été sensibilisées sur la PF.

La collaboration entre les deux secteurs a permis d'améliorer les procédures d'approvisionnement et de gestion des données. Avec la mise en œuvre du projet SMNI / FP / PALU, les systèmes d'approvisionnement et de logistique publics et privés sont désormais mieux intégrés. En outre, les prestataires privés transmettent désormais leurs données statistiques aux districts sanitaires et celles-ci sont prises en compte dans le système national d'information sanitaire. Cette collaboration a contribué à la mise en place d'un système de santé plus organisé et réactif. Ce partenariat a permis ainsi d'instaurer une meilleure collaboration entre la région médicale/les districts et les entreprises et cliniques qui sont désormais plus impliquées dans la mise en œuvre des activités sanitaires initiées par les régions emédicales et les districts sanitaires. Par ailleurs, la supervision des activités des entreprises et cliniques privées est intégrée dans la supervision de routine au niveau des régions médicales et districts. II a été également notée une certaine émulation entre les entreprises qui veulent demontrer qu'elles offrent un nouveau services, à savoir la PF, à leurs employés et leurs familles.

\section{Défis et Conclusion}

Au cours de la mise en œuvre de ce partenariat public-privé, il a fallu surmonter des défis. Certaines entreprises privées ont eu à faire face à des difficultés dans la fourniture de nouveaux services de santé tels que prévus initialement par le projet, ainsi qu'à des défis dans la consolidation de ces services. En effet, l'introduction de ces nouveaux services a occasionné des dépenses supplémentaires pour ces entreprises notamment celles engendrées par la nécessité de réorganiser les services. Un plaidoyer plus accru pourrait aider à renforcer la visibilité, l'utilisation et l'importance de la fourniture de ces services au niveau de ces entreprises.

Toutefois, il convient de noter qu'au Sénégal, la grande majorité des entreprises privées ciblées ont bien accueilli ces activités de sensibilisation novatrices, se sont engagées en faveur d'une meilleure gestion du système d'approvisionnement des produits contraceptifs et du système d'information sanitaire. Par ailleurs, elles ont fait preuve d'un intérêt général dans le soutien de ce partenariat en vue de l'atteinte des objectifs nationaux de santé. Les engagements du secteur privé au Sénégal devraient être constamment consolidés à travers la mise en place d'un environnement réglementaire durable et la signature de protocoles d'accord clairement définis. Avec la participation continue du secteur privé, le Sénégal a un plus grand potentiel pour améliorer les indicateurs dans le domaine de la santé de la reproduction.

Décembre 2010

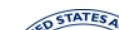

STATESAC

* $\frac{\text { USAID }}{\text { in }}$ प्रा110 ONALDEVI

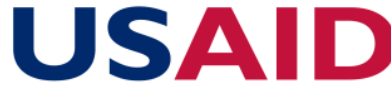
FROM THE AMERICAN PEOPLE

Ce résumé a été rendu possible grâce à l'appui apporté par le peuple américain à travers l'Agence Américaine Pour le Développement International

(USAID).Les contenus sont de la seule responsabilité des auteurs et ne reflètent pas nécessairement les vues de l'USAID ou du gouvernement des Etas Unis

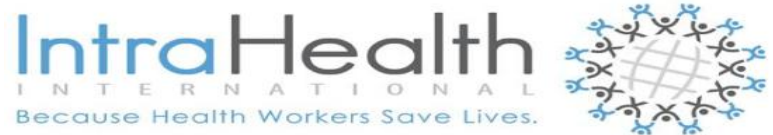

(f) Population Council 